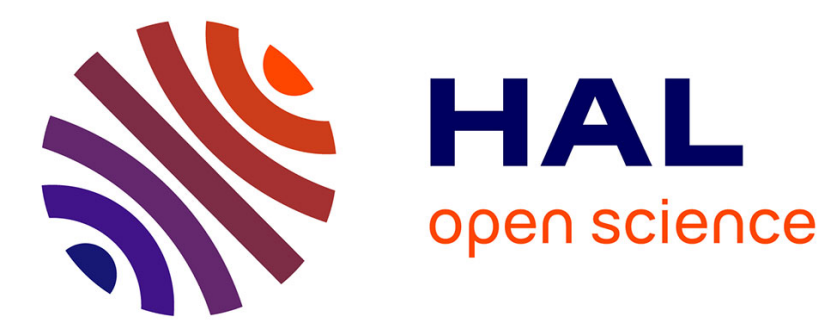

\title{
LE DESIGN MANAGEMENT EN DISCUSSION
}

Veronique Cova, Guillaume Blum

\section{To cite this version:}

Veronique Cova, Guillaume Blum. LE DESIGN MANAGEMENT EN DISCUSSION. Sciences du Design, 2018. hal-02062215

\section{HAL Id: hal-02062215 https://hal.science/hal-02062215}

Submitted on 19 Mar 2019

HAL is a multi-disciplinary open access archive for the deposit and dissemination of scientific research documents, whether they are published or not. The documents may come from teaching and research institutions in France or abroad, or from public or private research centers.
L'archive ouverte pluridisciplinaire HAL, est destinée au dépôt et à la diffusion de documents scientifiques de niveau recherche, publiés ou non, émanant des établissements d'enseignement et de recherche français ou étrangers, des laboratoires publics ou privés. 


\section{Ouverture}

\section{LE DESIGN MANAGEMENT EN DISCUSSION}

Guillaume Blum, Véronique Cova

Presses Universitaires de France | «Sciences du Design »

2018/1 n ${ }^{\circ} 7$ | pages 21 à 27

ISSN 2428-3711

ISBN 9782130803225

Article disponible en ligne à l'adresse :

https://www.cairn.info/revue-sciences-du-design-2018-1-page-21.htm

Pour citer cet article :

Guillaume Blum, Véronique Cova « Le design management en discussion », Sciences du Design 2018/1 (n 7), p. 21-27.

Distribution électronique Cairn.info pour Presses Universitaires de France.

(C) Presses Universitaires de France. Tous droits réservés pour tous pays.

La reproduction ou représentation de cet article, notamment par photocopie, n'est autorisée que dans les limites des conditions générales d'utilisation du site ou, le cas échéant, des conditions générales de la licence souscrite par votre établissement. Toute autre reproduction ou représentation, en tout ou partie, sous quelque forme et de quelque manière que ce soit, est interdite sauf accord préalable et écrit de l'éditeur, en dehors des cas prévus par la législation en vigueur en France. Il est précisé que son stockage dans une base de données est également interdit. 


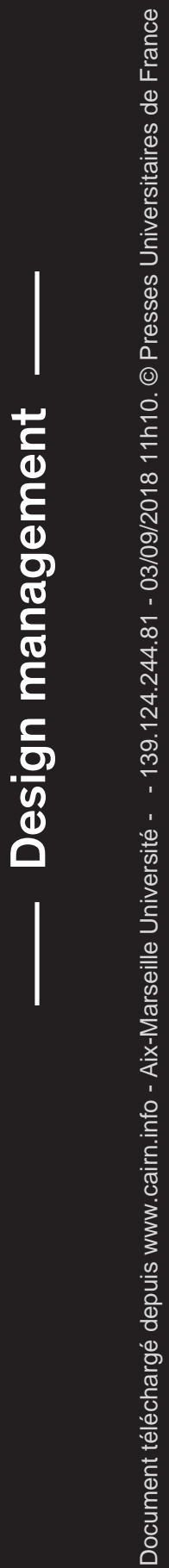




\section{Ouverture}

\section{Guillaume Blum}

Professeur à l'École de design de l'Université Laval

Groupe de recherche Design, innovations et humanismes

Centre interuniversitaire de recherche

sur la science et la technologie (CIRST)

guillaume.blum@design.ulaval.ca

\section{Véronique Cova}

Professeur des Universités

Graduate School of Management - IAE

Aix-Marseille Université

Centre d'Études et de Recherche en Gestion

d'Aix-Marseille (CERGAM)

veronique.cova@iae-aix.com 


\section{Le design management \\ en discussion}

Initialement centré sur ses domaines traditionnels (design industriel, de produits, design graphique), le design a, avec le temps, élargi sa base d'intervention à d'autres activités. D'abord aux services, puis à l'organisation elle-même. Ainsi, depuis déjà plusieurs années, le concept de design management fait partie des pratiques observées sur le terrain et constitue un champ de recherche. À titre d'exemple, le magazine américain Fast Compagny titrait en octobre 2016 en couverture un dossier d'une trentaine de pages sur le thème Innovation by design - how to disrupt business as usual (un des thèmes importants du design management). On note également l'émergence avec le temps de plusieurs organisations et institutions réunissant professionnels et/ou chercheurs (Design management institute, Design management academy, Design management review, Design management journal, etc.). En France, les travaux de Brigitte Borja de Mozota - dont on retrouve un article dans le cadre de ce numéro - ont défriché le territoire disciplinaire dès les années 1990. Plus de 30 ans après l'émergence des premiers travaux, qu'en est-il aujourd'hui du design management?

L'objectif de ce dossier thématique est de dresser un portrait du design management en 2018.

II semble difficile de regrouper ensemble ces deux termes, et nombreux sont ceux qui sentent les divergences entre le management et le design. Pour simplifier, le management est généralement orienté vers les questions d'efficience, là où le design se tourne vers celles touchant à la conception créative. On retrouve ici le dilemme entre les rôles d'exploration et d'exploitation tels que posés par March (1991). Pourtant, les approches centrées sur la gestion de l'innovation et des connaissances tendent à montrer qu'elles ne s'opposent pas nécessairement. Ainsi, l'activité innovante, génératrice de savoir, est souvent à la source d'une plus grande efficacité. Mais aussi, une approche trop centrée sur les indicateurs d'efficacité est une approche planant au-dessus du réel sans en comprendre les enjeux (Dujarier, 2015). En fait, le design et le management, tel que chacun devrait être exercé, se rencontrent dans 
leur approche concrète, reliée au réel, à l'industrie, à la nécessité de prendre en considération les contraintes de production, les contraintes de consommation, dans des contextes interdisciplinaires.

Au même titre que le designer n'est ni un ingénieur, ni un artiste, il n'est pas un gestionnaire ou un marketeur. L'hybridité de son profil n'empêche nullement une spécificité qui lui est propre et qui, en fait, constitue son cœur de métier. De la même façon, le design ne devrait appartenir ni aux sciences de la conception, ni aux sciences de l'art, ni aux sciences de gestion, même si la tentation de rapatriement est forte par chacun des domaines scientifiques concernés. Le design devrait appartenir aux sciences du design, car les projets scientifiques, les épistémologies, les acteurs, les méthodes et les outils restent différents. Fondamentalement interdisciplinaires, les sciences du design doivent prendre en considération les aspects sociaux, psychologiques, ergonomiques, techniques, esthétiques, organisationnels. Certes, tout comme le marketing qui se positionne comme "orienté consommateur», ou l'ingénierie IHM (Interface Humain-Machine) qui interroge les interfaces, le design défend une centralité des usages et par là, de l'usager. Mais ce n'est pas une raison suffisante pour assimiler l'un à l'autre, ne serait-ce que par les spécificités de leurs méthodes d'interrogation du réel.

Le design management n'est donc pas une bidiscipline; ce n'est ni le management du design ni le design comme une nouvelle forme de management. S'il caractérise la tentation de rapatriement citée ci-dessus, il ne trouverait sa place que dans l'humilité de chaque partie. Or l'histoire du design management témoigne d'une volonté de conquête de champs.

Ainsi, un maillage nous semble utopique et contreproductif, même s'il représente le pas d'après une juxtaposition. Ni l'un ou ni l'autre, ni à la fois l'un et l'autre. Il est plus aisé de dire ce que le design management n'est pas. Il est plus difficile de préciser ce qu'il est. Contentons-nous ici d'approcher ce que pourrait être sa place.

Le design management n'est pas un territoire; c'est une relation entre deux territoires, le management et le design. II est un pont jeté entre eux et en cela possède son propre territoire, le territoire de l'entredeux, le territoire du possible revisité qui ose se jouer des frontières et proposer un espace de pensée empreint d'hybridité et de reliance ${ }^{01}$. Ce disant, nous avons parfaitement conscience de la variabilité et de la fluidité du design management.

Alors quoi? Aux designers de faire de la recherche en design. Il en va de la formalisation des frontières et du territoire des sciences du design. Et du design management. II nous semble, au vu des avancées disciplinaires, qu'un travail important de théorisation reste à faire et notamment d'un point de vue épistémologique. Pour que progresse la pratique, le design management a besoin de se placer, de se justifier, d'être reconnu, et donc qu'une plus grande pratique implique au préalable un plus grand travail de conceptualisation. II s'agit ainsi de prendre une distance salutaire avec l'usage d'outils, processus et techniques intégrant des valeurs et représentations du monde (Berry, 1983) pour mener une réflexion préalable à ces valeurs et représentations du monde. 
De même qu'on n'a pas idée d'un chercheur en gestion qui n'aurait jamais mis les pieds dans une entreprise, ou un ingénieur qui ne connaitrait aucune machine, ni même encore, et pour sortir du champ, d'un chercheur en médecine qui n'aurait jamais vu un malade, il n'est pas concevable d'envisager un chercheur en design qui ne se soit pas frotté au design lui-même. L'approche disciplinaire du design comme un domaine scientifique à part entière se confronte à deux difficultés: dans sa relation aux autres disciplines d'une part; dans sa relation à elle-même d'autre part. Le passage de la pratique à la science demande aux designers d'interroger leur mise en projet qui, elle, se situe au sein du tissu socioéconomique. Il devient ainsi plus pertinent que jamais de considérer les organisations non pas comme une fin, mais comme un moyen permettant de mener à bien la recherche en design.

Ce dossier thématique vise à faire le point sur les enjeux du design management. II reflète ce fort ancrage dans la réalité et l'analyse des travaux scientifiques existants. Tout d'abord, en revenant sur l'historique du design management, sur les concepts clés développés à travers le temps, sur l'évolution des pratiques et des discours. En dressant un portrait de la situation actuelle, de son champ d'activité, de son usage réel et des avancées théoriques et pratiques réalisées. Finalement, en traitant du design management dans une optique prospective, afin de dégager les pistes futures pour les praticiens et les chercheurs. Car ce dossier ne se veut pas être un point final, mais plutôt un point d'étape pour la suite de la recherche. II s'agit donc de réaliser un portrait réaliste du design management en 2018.

Les trois contributions qui constituent cet important dossier permettent de nourrir le débat. Trois articles que l'on pourrait voir comme un retour réflexif sur le passé avec l'étude des travaux marquants en design management (Borja de Mozota), comme une analyse du tissu économique actuel des organisations avec l'établissement d'un outil de diagnostic pour les petites et moyennes entreprises (Glaubert et al.), comme ancré dans des entreprises pour déterminer dans le futur les dynamiques organisationnelles et stratégiques que permet le design (Berger et al.). Ces trois articles sont riches en pensées et ouvrent de belles perspectives de recherche.

L'article de Brigitte Borja de Mozota constitue une analyse des travaux marquant le champ du design management depuis les premiers travaux. Analyse réflexive sur quarante années de recherches et de pratiques, cet article donne un éclairage nouveau venant compléter le travail d'Erichsen et Christensen (2013). Il vise à rendre visible le design en dehors du design, et notamment l'intérêt d'approches hybrides entre le design et le management. Ce que cet article présente remarquablement, c'est la dynamique d'évolution disciplinaire du design management. Ainsi, l'auteure montre le passage du design de rôle périphérique comme un outil support, à une fonction de l'entreprise pour devenir depuis une dizaine d'années une fonction transverse de l'entreprise. L'auteure présente sur trois périodes de temps (1975-1992; 1993-2005; 20062017) l'évolution de plusieurs concepts clés du design management: la valeur, les méthodes et compétences, les outils, l'intégration avec 
les autres fonctions, le rôle de leadership. Cela amène à une réflexion sur les liens entre design et management, les interactions et la nécessité d'une réflexion conjointe entre recherche et pratique. À partir de cette analyse historique, l'auteure formule des pistes d'analyse pour le futur du design management.

L'article de Daphna Glaubert, Nathalie Nyffeler et Luc Bergeron présente le développement d'un outil d'audit du design management, appliqué et applicable aux Petites et Moyennes Entreprises, acteurs trop souvent mis à l'écart de la recherche et des politiques économiques. Le travail présenté est basé sur une analyse de la littérature et sur de la recherche-action en interaction avec des praticiens et entreprises. Les trois auteur-e.s mettent ainsi en œuvre leurs connaissances théoriques et empiriques pour élaborer un outil de diagnostic intéressant présenté sous la forme d'une cartographie. Cette dernière inclut 19 sous-thématiques regroupées en 5 leviers: processus, marque, relation client, offres et stratégie. L'intérêt de l'outil développé est multiple et permet, entre autres choses, d'établir un diagnostic, donc d'identifier des objectifs d'amélioration. II facilite la réflexivité des acteurs et le dialogue entre eux. II amène à mieux saisir la complexité d'une approche reposant sur le design management tout en donnant des leviers d'action. Finalement, l'outil cartographique sert lui-même d'objet-frontière favorisant l'échange entre individus et entités (groupe de travail, équipe projet, département, etc.).

Finalement, Estelle Berger, lona Ocnarescu et Frédérique Pain nous présentent le premier opus d'une série d'articles sur la mise en œuvre de leur projet de recherche. Celui-ci vise à étudier sur le terrain et sur la durée, dans plusieurs entreprises, la transformation managériale et économique des organisations sous l'influence du design. Il s'agit plus spécifiquement d'étudier comment les dynamiques du design permettent l'amélioration de l'expérience et la mise en œuvre de stratégies pour les entreprises participantes au sein du laboratoire Exalt. Dans l'article, les auteures reviennent sur les débuts du projet pour en exposer les prémices, ce qui permettra à terme un travail d'archéologie du savoir, et d'analyse de la trajectoire des acteurs. Les chercheures font ressortir plusieurs points importants: l'imbrication des niveaux individuel, organisationnel et écosystémique; l'importance du travail au sein d'une communauté épistémique; le besoin d'un allié au sein des organisations étudiées, que constitue le boundary spanner lequel permet de transcender les frontières fonctionnelles et disciplinaires. Nul doute que ce projet d'ampleur, tourné vers le futur, permettra d'ici quelques années d'améliorer la compréhension de la dimension stratégique du design au sein des organisations.

Nous espérons que vous prendrez autant de plaisir à découvrir et partager ces recherches que nous en avons eu à les sélectionner. Elles marquent une avancée notable dans la définition et la compréhension du design management. 
NOTES

01.

Selon Edgar Morin, la notion de reliance est le substantif inventé qui manquait à l'adjectif. " "Relié” est passif, "reliant" est participant, "reliance" est activant. On peut parler de "déliance" pour l'opposé de "reliance"” (Morin, 2004, p. 239).

\section{RÉFÉRENCES}

BERRY, M. (1983). Une technologie invisible. L'impact des instruments de gestion sur l'évolution des systèmes humains. Paris: CRG, École polytechnique. Repéré à l'adresse https://hal.archivesouvertes.fr/hal-00263141/

DUJARIER, M.-A. (2015).

Le management désincarné. Paris: La Découverte.

ERICHSEN, P.G., ET CHRISTENSEN, P.R. (2013). The evolution of the design management field: a journal perspective. Creativity and innovation Management, 22 (2), 107-120.

MARCH, J. G. (1991). Exploration and Exploitation in Organizational Learning. Organization Science, 2(1), 71-87.

MORIN, E. (2004). La méthode, Volume 6 : Éthique. Paris: Le Seuil. 